\title{
Compliance With Weight-bearing Precautions: A Prospective Observational Study
}

\section{Michael Murphy ${ }^{1}$, Bailey Johnson ${ }^{1}$, William Davis ${ }^{1}$, Dimitri Mabarak ${ }^{1}$, Hobie Summers ${ }^{1}$, Joseph Cohen ${ }^{1}$, William Lack ${ }^{2}$}

1. Loyola University Medical Center, Stritch School of Medicine, Department of Orthopaedic Surgery and Rehabilitation, Maywood, Illinois, USA 2. Harborview Medical Center, University of Washington School of Medicine, Department of Orthopaedic Surgery and Rehabilitation, Seattle, Washington, USA

\section{INTRODUCTION}

- Patients are instructed on weight-bearing restrictions after orthopaedic trauma based on historical norms, clinical intuition, and biomechanical research.

- There exists a paucity of information of how compliance varies by weight-bearing instruction.

\section{PURPOSE}

- To identify differences in compliance between weight-bearing instructions.

- To identify questions, patient demographics, and injury characteristics that the surgeon may use to predict compliance.

\section{METHODS}

- We prospectively identified forty-five (45) lower extremity trauma patients (27 male and 18 female).

- Weight-bearing instructions included:

- Non-weight-bearing (NWB, $n=30$ )

- Touchdown 30 pounds weight-bearing (TDWB, $\mathrm{n}=11$ )

- As tolerated (WBAT, $n=4)$

- Weight-bearing compliance was determined with Strydalyzer INSIGHT insole pressure sensors during inpatient stay $(n=45)$ and at 2week ( $n=34)$ follow-up.

- Patients completed questionnaires at their inpatient stay.

- Patients were given pedometers to wear at home at all times.

- Patient age, gender, weight, educational level, fracture patters, step count on home pedometer, Timed Up and Go, Visual Analog Scale pain score, Charlson Comorbidity Index (CCI), American Society of Anesthesiologists (ASA) score, drug use, distance ambulated during inpatient stay, disposition upon discharge, number of therapy visits, average caloric expenditure prior to injury, handedness, concurrent injuries, and grip strength (as measured by a dynamometer).

\section{RESULTS}

- Mean age was 47.7 (range: 21 to 84 ) years old.

- Fractures:

- Pelvis: $n=5$

- Femur: $\mathrm{n}=11$

- Tibia: $n=26$

- Foot: $n=3$

- Inpatient noncompliance was statistically associated with pre-injury exhaustion $(p<0.02)$.

Noncompliance at 2 week follow-up was associated with TDWB status $(p<0.01)$, difficulty ascending 12 stair flights prior to injury $(p=0.02)$, inpatient disorientation $(p<0.01)$, poor recall as measured by the Mini Mental Status Exam $(p<0.05)$, and a lesser distance ambulated during inpatient stay $(p<0.01)$.

Median (maximum) weight borne

- During inpatient stay:

- WBAT: 100 (163) lbs

- TDWB: 29 (191) lbs

- NWB: 0 (99) lbs

At 2 week follow-up:

- WBAT: 137 (191) lbs

- TDWB: 29 (154) lbs

- NWB: 0 (12) lbs

Within the TDWB group, the median (range) weight borne by those exceeding instructions was:

- During inpatient stay: 177 (117 to 191) lbs

- At 2 week follow-up: 117 (84 to 154) lbs

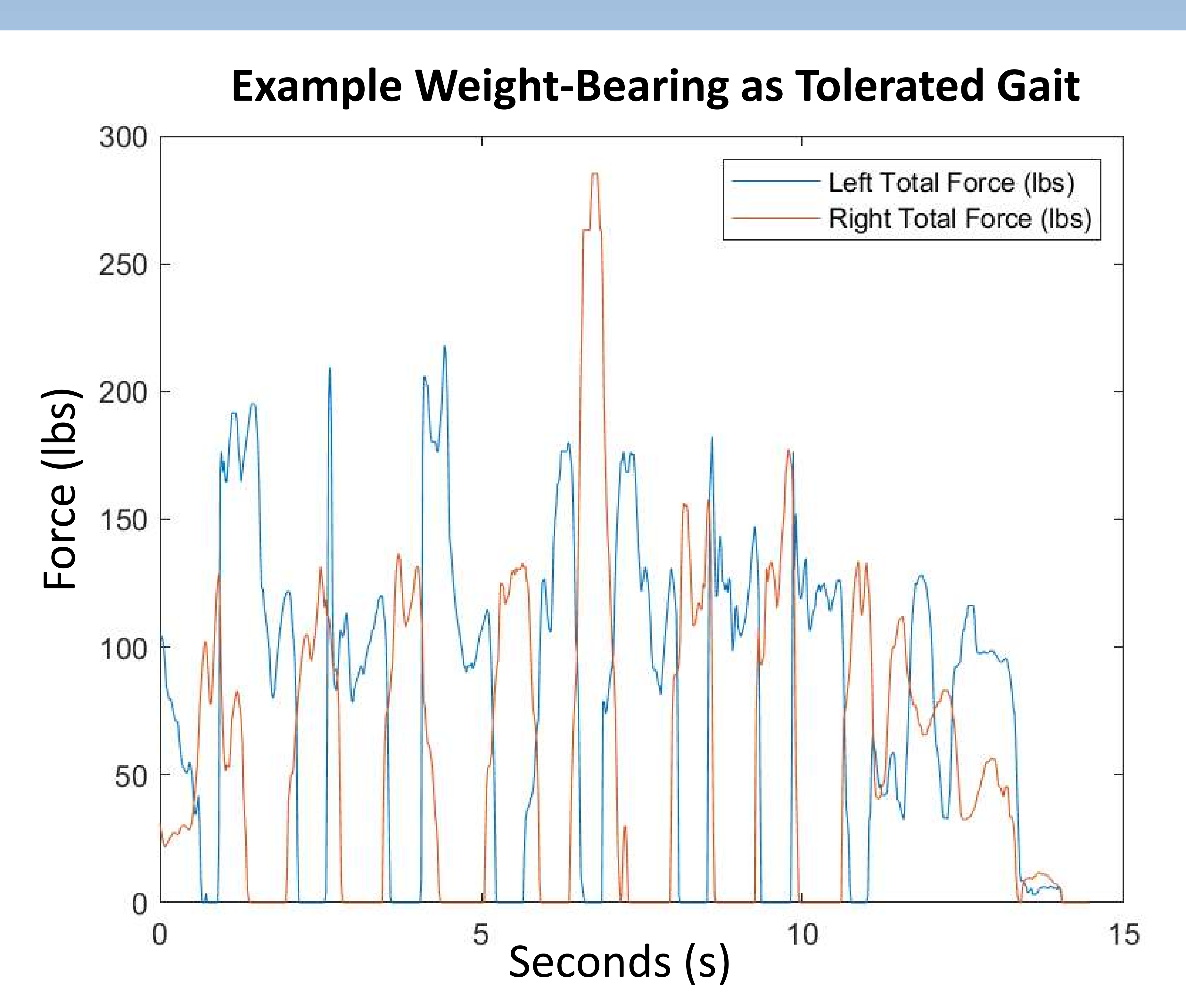

Example Touchdown Weight-Bearing Gait
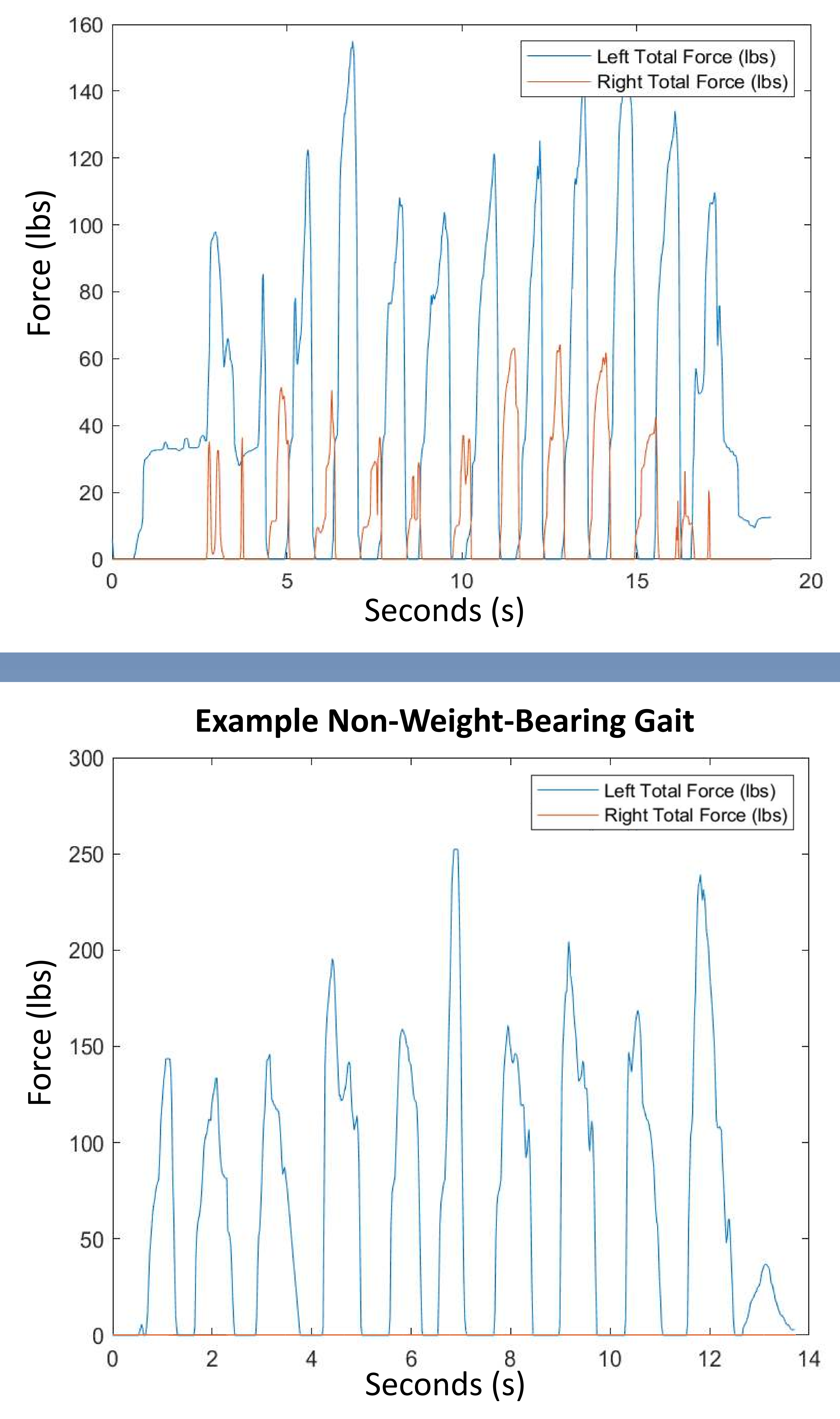

DISCUSSION / CONCLUSIONS

- Inpatient compliance was similar for different restrictions.

- However, TDWB instructions were associated with postdischarge noncompliance.

- This was most likely to occur in the setting of frailty and mental limitations.

- We recommend such patients be best treated with dichotomization to either NWB or WBAT. 